\title{
Thymidine phosphorylase mRNA expression may be a predictor of response to post-operative adjuvant chemotherapy with S-1 in patients with stage III colorectal cancer
}

\author{
MASAICHI OGAWA, MICHIAKI WATANABE, YOSHINOBU MITSUYAMA, TADASHI ANAN, \\ MASAHISA OHKUMA, TETSUYA KOBAYASHI, KEN ETO and KATSUHIKO YANAGA \\ Department of Surgery, The Jikei University School of Medicine, Tokyo 105-8461, Japan
}

Received February 12, 2013; Accepted August 12, 2014

DOI: $10.3892 / 01.2014 .2574$

\begin{abstract}
The aim of the present study was to investigate markers in surgically resected specimens of colorectal cancer that can be used to predict the response to chemotherapy. The mRNA expression levels of enzymes involved in 5-fluorouracil (5-FU) metabolism and folate metabolism were measured in formalin-fixed, paraffin-embedded tumor sections obtained from the primary tumors of 54 patients with resected stage II or III colorectal cancer who received S-1 for one year. The 5-FU metabolizing enzymes studied were thymidylate synthase, dihydropyrimidine dehydrogenase and thymidine phosphorylase (TP). The folate metabolizing enzymes studied were folypolyglutamate synthetase, $\gamma$-glutamyl hydrolase and dihydrofolate reductase. The associations between the mRNA expression levels of these enzymes and clinical variables were investigated. Tumors were classified as exhibiting high or low expression as compared with the median mRNA expression level of each metabolizing enzyme defined as the cutoff value. The associations between the high and low expression levels of each enzyme and disease-free survival (DFS) were analyzed with the use of Kaplan-Meier curves and the log-rank test. DFS was not significantly associated with the relative mRNA expression level of any metabolizing enzyme in the study group as a whole, but there was a trend toward longer DFS in patients with high TP expression $(\mathrm{P}=0.066)$. In patients with stage III colorectal cancer, high TP expression was associated with significantly improved outcomes compared with low TP expression $(\mathrm{P}=0.039)$. These results indicate that the mRNA expression of TP, a metabolizing enzyme of 5-FU, is
\end{abstract}

Correspondence to: Dr Masaichi Ogawa, Department of Surgery, The Jikei University School of Medicine, 3-25-8 Nishi Shinbashi, Minato-ku, Tokyo 105-8461, Japan

E-mail: masatchmo1962@yahoo.co.jp

Key words: thymidine phosphorylase, S-1, adjuvant chemotherapy, colorectal cancer a significant predictor of response to post-operative chemotherapy with S-1 in patients with stage III colorectal cancer.

\section{Introduction}

5-Fluorouracil (5-FU) continues to play a central role in chemotherapy for colorectal cancer. S-1 is an oral 5-FU-based anticancer drug that was developed in Japan. This drug combines tegafur, a prodrug of 5-FU, with gimeracil, a reversible antagonist of the rate-limiting enzyme of the metabolic pathway of 5-FU, and oteracil potassium, which is distributed at high concentrations in the gastrointestinal tract, where it reduces gastrointestinal toxicity by irreversibly inhibiting the phosphorylation of 5-FU. The molar ratio of tegafur, gimeracil and oteracil potassium in $\mathrm{S}-1$ is 1.0:0.4:1.0 (1,2). Clinically, the time course of serum 5-FU concentrations following the oral administration of S-1 has been confirmed to be similar to that during a continuous infusion of 5-FU (3). Late phase II studies of patients with advanced or recurrent colorectal cancer have reported a response rate of $37.4 \%$ in patients with advanced or recurrent colorectal cancer $(4,5)$. As a second-line chemotherapy for metastatic colorectal cancer, combination therapy with S-1 and irinotecan was shown to be non-inferior to a folinic acid, 5-FU and irinotecan (FOLFIRI) regime (6). Clinical trials have demonstrated that administering S-1 for one year is as effective as post-operative adjuvant chemotherapy in stage II or III gastric cancer. Together with the results of our previous feasibility study, this suggests that $\mathrm{S}-1$ is a promising drug for adjuvant therapy in patients with stage II or III colorectal cancer (7-9).

Recent studies have provided evidence that the expression of enzymes involved in 5-FU metabolism is associated with treatment response $(10,11)$. Thymidylate synthase (TS), a rate-limiting enzyme in DNA synthesis, dihydropyrimidine dehydrogenase (DPD), an enzyme participating in the catabolism of 5-FU, and thymidine phosphorylase (TP), an important metabolizing enzyme of 5-FU, have been studied as predictors of the response or sensitivity to anticancer agents. Enzymes involved in folate metabolism, including folypolyglutamate synthetase (FPGS), $\gamma$-glutamyl hydrolase (GGH) and dihydrofolate reductase (DHFR), have been reported to participate in 
the response to 5-FU-based agents. S-1 is an oral 5-FU-based anticancer drug for which it is considered meaningful to study the value of enzymes involved in 5-FU metabolism or folate metabolism as predictors of treatment response or drug sensitivity.

In the present study, the mRNA expression levels of enzymes involved in 5-FU metabolism or folate metabolism were measured, using tumor specimens obtained from patients with stage II or III colorectal cancer who received post-operative adjuvant chemotherapy with $\mathrm{S}-1$ for one year. The aim of the study was to assess the value of such expression levels as predictors of the response or sensitivity to S-1.

\section{Materials and methods}

Patients and treatment. The study group was comprised of patients in whom stage II or III colorectal cancer was diagnosed (Union for International Cancer Control staging, sixth edition) (12) and curatively resected in The Jikei University School of Medicine between February 2004 and June 2006. Patients received oral S-1 (Taiho Pharmaceutical Co., Ltd., Tokyo, Japan) as post-operative adjuvant chemotherapy. The daily dose of S-1 (80, 100 or $120 \mathrm{mg}$ per day) was calculated according to body-surface area and administered in two divided doses, one after breakfast and the other after dinner, for 28 consecutive days, followed by a 14-day rest period. The dose of $\mathrm{S}-1$ was $80 \mathrm{mg} /$ day if the body-surface area was $<1.25 \mathrm{~m}^{2}, 100 \mathrm{mg} /$ day if the body-surface area was 1.25 to $<1.5 \mathrm{~m}^{2}$ and $120 \mathrm{mg}$ /day if the body-surface area was $\geq 1.5 \mathrm{~m}^{2}$. The duration of treatment with $\mathrm{S}-1$ was one year. This study was approved by the ethical committee of The Jikei University School of Medicine (Tokyo, Japan) and written informed consent was obtained from all patients.

\section{Laboratory analysis}

Expression. Tumor tissue expression levels of various metabolizing enzymes were measured using pathological specimens of resected colorectal cancer. Gene mRNA expression levels of the TS, DPD, TP, FPGS, GGH and DHFR enzymes were semiquantitatively measured by the Dannenberg tumor profiling (DTP) method, and the associations between such levels and clinical variables were studied. An outline of the Dannenberg tumor profiling method is presented below.

Staining of formalin-fixed, paraffin-embedded (FFPE) tissue sections. To confirm the site of the tumors, $5-\mu \mathrm{m}$ thick FFPE tissue sections were stained with hematoxylin and eosin (HE), and the site of cancer was identified and marked by a pathologist. Additionally, $10-\mu \mathrm{m}$ thick FFPE tissue sections were stained with nuclear fast red (NFR) for RNA extraction.

Slicing of cancer tissue. Cancer tissue within the tumor, as designated by a pathologist on examination of specimens stained with NFR under a stereomicroscope, was thinly sliced with a razor knife, surgical knife or laser microdissector, and the slices were placed in RNA extraction buffer. Usually, an area $>50 \mathrm{~mm}^{2}$ was shaved to maintain at least $80 \%$ cancer cells. Cancer cells were stained a darker red by NFR compared with the normal cells. Cancer tissue was cut out on the basis of the staining pattern, and specimens stained with HE served as a reference.
RNA extraction and circular DNA (cDNA) synthesis. Proteinase was added to a cancer-cell suspension, and the mixture was heated to cause cytolysis. RNA was refined by simple column extraction or by phenol extraction and ethanol precipitation, and cDNA synthesis was synthesized using random hexamer as a primer.

Analysis by quantitative reverse transcription $(R T)$ polymerase chain reaction $(P C R)$. Formalin-fixed $10-\mu \mathrm{m}$ thick paraffinembedded sections of resected primary colorectal cancer tumors were obtained from identified areas with the highest tumor concentration and were then mounted onto uncoated glass slides. For histological diagnosis, representative sections were stained with haematoxylin and eosin using standard methods. Prior to microdissection, sections were stained with nuclear fast red (American MasterTech, Lodi, CA, USA). The sections were selectively isolated by laser capture microdissection (P.A.L.M. Microsystem; Leica, Wetzlar, Germany), according to standard procedures (13). The dissected tissues were transferred to a reaction tube containing $400 \mu \mathrm{l}$ RNA lysis buffer (Invitrogen Life Technologies, Carlsbad, CA, USA).

The samples were homogenised at $92^{\circ} \mathrm{C}$ for $30 \mathrm{~min}$. A total of $50 \mu \mathrm{m}$ of $2 \mathrm{M}$ sodium acetate ( $\mathrm{pH} 4.0$ ) was added, followed by $600 \mu \mathrm{l}$ phenol/chloroform/isoamyl alcohol (250:50:1). The tubes were vortexed for $15 \mathrm{sec}$, placed on ice for $15 \mathrm{~min}$, and then centrifuged at $16,000 \mathrm{x} \mathrm{g}$ for $8 \mathrm{~min}$ at $8^{\circ} \mathrm{C}$ centrifuge. The upper aqueous phase was removed and placed in a $1.5 \mathrm{ml}$ centrifuge tube. A total of $10 \mu \mathrm{l}$ glycogen and 300-400 $\mu \mathrm{l}$ isopropanol were added and the samples were vortexed for 10-15 sec. The tubes were chilled at $-20^{\circ} \mathrm{C}$ for $30-45 \mathrm{~min}$ to precipitate the RNA. The samples were then washed in $500 \mu \mathrm{l}$ $75 \% \mathrm{v} / \mathrm{v}$ ethanol and air-dried for $15 \mathrm{~min}$. The pellet was resuspended in $50 \mu 15 \mathrm{mM}$ Tris buffer (Sigma-Aldrich, St. Louis, MO, USA). Finally, cDNA was prepared as described by Lord et al (14). Quantification of the 12 genes of interest and an internal reference gene ( $\beta$-actin) was performed using a fluorescence-based real-time detection method (ABI PRISM 7900 Sequence Detection System; Applied Biosystems, Foster City, CA, USA). The PCR reaction mixture consisted of $120 \mathrm{nM}$ of each primer, $200 \mathrm{nM}$ probe, $0.4 \mathrm{U} / 1$ of AmpliTaq gold polymerase, $200 \mathrm{nM}$ of each dATP, dCTP, dGTP, dTTP, $3.5 \mathrm{mM} \mathrm{MgCl}_{2}$ and $1 \times$ Taqman buffer, containing a reference dye (Applied Biosystems). The final volume of the reaction mixture was $20 \mu \mathrm{l}$. PCR conditiosn were as follows: $50^{\circ} \mathrm{C}$ for $2 \mathrm{~min}$ and $95^{\circ} \mathrm{C}$ for $10 \mathrm{~min}$, followed by 46 cycles of $95^{\circ} \mathrm{C}$ for $15 \mathrm{sec}$ and $60^{\circ} \mathrm{C}$ for $1 \mathrm{~min}$. The primers and probe used were as follows: Forward, 5'-GCCTCGGTGTGCCTTTCA-3' and reverse, 5'-CCCGTGATGTGCGCAAT-3' for TS; and Taqman probe 5'-TCGCCAGCTACGCCCTGCTCA-3'; and forward 5'-AGGACGCAAGGAGGGTTTG-3' and reverse, 5'-GTCCGCCGAGTCCTTACTGA-3' for DPD; and Taqman probe 5'-CAGTGCCTACAGTCTCGAGTCTGCCAGT-3'; forward, 5'-CCTGCGGACGGAATCCT-3' and reverse, 5'-GCTGTGATGAGTGGCAGGCT-3'for TP; and Taqman probe 5'-CAGCCAGAGATGTGACAGCCACCGT-3'; foward, 5'-GGCTGGAGGAGACCAAGGAT-3' and reverse, 5'-CATGAGTGTCAGGAAGCGGA-3' for FPGS; and Taqmanprobe 5'-CAGCTGTGTCTCCATGCCCCCCTAC-3'; forward, 5'-GCGAGCCTCGAGCTGTCTA-3' and reverse, 
Table I. Patient characteristics.

\begin{tabular}{|c|c|}
\hline Parameter & Value \\
\hline \multicolumn{2}{|l|}{ Age, years } \\
\hline Median (range) & $67(31-84)$ \\
\hline \multicolumn{2}{|l|}{ Gender, n (\%) } \\
\hline Male & $39(72.2)$ \\
\hline Female & $15(27.8)$ \\
\hline \multicolumn{2}{|l|}{ Primary site, $\mathrm{n}(\%)$} \\
\hline Colon/rectosigmoid & $40(74.1)$ \\
\hline Rectum & $14(25.9)$ \\
\hline \multicolumn{2}{|l|}{ Invasion depth, n (\%) } \\
\hline MP & $1(1.9)$ \\
\hline SM & $1(1.9)$ \\
\hline SS & $21(38.9)$ \\
\hline SE & $14(25.9)$ \\
\hline SI & $4(7.4)$ \\
\hline A & $13(24.1)$ \\
\hline \multicolumn{2}{|c|}{ Lymph node metastasis, n (\%) } \\
\hline No & $16(29.6)$ \\
\hline N1 & $23(42.6)$ \\
\hline $\mathrm{N} 2$ & $12(22.2)$ \\
\hline N3 & $3(5.6)$ \\
\hline \multicolumn{2}{|l|}{ Stage, n (\%) } \\
\hline II & $16(29.6)$ \\
\hline III & $38(70.4)$ \\
\hline
\end{tabular}

MP, muscularis propria; SM, submucosa; SS, subserosa; SE, serosal exposure; SI, serosal invasion; A, adventitia.

Table II. Expression level of mRNA $(n=54)$.

\begin{tabular}{lr}
\hline Molecular markers & \multicolumn{1}{c}{ Median (range) } \\
\hline TS & $4.075(1.100-20.48)$ \\
DPD & $0.295(0.050-1.080)$ \\
TP & $2.575(0.880-23.94)$ \\
FPGS & $0.590(0.220-1.370)$ \\
GGH & $12.961(1.600-166.5)$ \\
DHFR & $5.380(1.620-12.53)$ \\
\hline
\end{tabular}

mRNA expression is relative to $\beta$-actin expression levels. TS, thymidylate synthase; DPD, dihydropyrimidine dehydrogenase; TP, thymidine phosphorylase; FPGS, folypolyglutamate synthetase; GGH, $\gamma$-glutamyl hydrolase; DHFR, dihydrofolate reductase.

5'-AATATTCCGATGATGGGCTTCTT-3' for GGH; and Taqman probe 5'-ACCCCACGGCGACACCGC-3'; forward, 5'-GTCCTCCCGCTGCTGTCA-3' and reverse, 5'-GCCGATGCCCATGTTCTG-3' for DHFR; and Taqman probe 5'-TTCGCTAAACTGCATCGTCGCTGTGTC-3'.

Calculation of results (DTP values). Genes are amplified two-fold on every cycle of PCR. Gene expression values

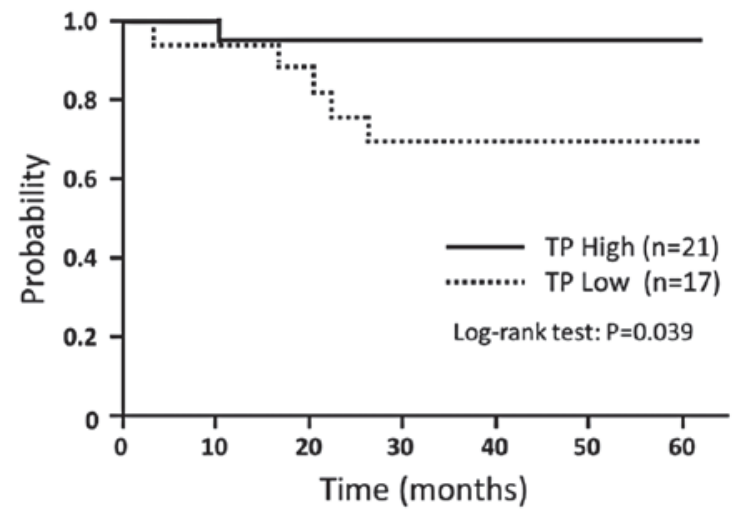

Figure 1. Kaplan-Meier plot of disease-free survival for stage III colorectal cancer patients according to thymidine phosphorylase (TP) expression level.

(relative mRNA levels) are expressed as ratios (differences between $\mathrm{Ct}$ values) of the gene of interest and the internal reference gene ( $\beta$-actin). Therefore, the gene expression ratio of each sample is calculated as a $2-\mathrm{Ct}$ value. The quantity of cancer cells removed from each specimen differs and was therefore expressed relative to the expression of $\beta$-actin expression to correct for differences in cell quantities. In addition, correction coefficients were calculated on the basis of the results of the analysis of standard samples containing known concentrations of target genes. The measured values were multiplied by the correction coefficients to derive DTP values, which were regarded as expression levels of the target genes. DTP values were calculated by the following formula, in which $\mathrm{dCt}$ is the $\mathrm{Ct}$ value of the target gene minus the $\mathrm{Ct}$ value of $\beta$-actin, and $\mathrm{K}$ is the correction coefficient: $\mathrm{DTP}=\mathrm{K} \times 2^{-\mathrm{dCt}}$.

Statistical methods. Disease-free survival (DFS), measured as the interval from the date of surgery to the date of the first documented evidence of recurrence, death or a second cancer, was calculated using the Kaplan-Meier method. The associations between the mRNA expression levels of the various metabolizing enzymes and the clinicopathological factors of age, gender, invasion depth, lymph-node metastasis, disease stage and tumor location were tested by Wilcoxon's test. The median mRNA expression level of each metabolizing enzyme was regarded as the cutoff value, and tumors were classified as having high or low expression as compared with this value. The associations between the high and low expression levels of each enzyme and DFS were analyzed using Kaplan-Meier curves, and differences between survival curves were computed with the log-rank test. $\mathrm{P}<0.05$ was considered to indicate a statistically significant difference.

\section{Results}

Patient characteristics. Between February 2004 and June 2006, a complete resection with no microscopically residual tumor (R0) was performed in 54 patients, who subsequently received oral S-1. The mRNA levels of the tumors were measured. Table I shows the demographic characteristics of the patients. The median age was 67 years (range, 31-84 years). The primary lesion was located in the 
Table III. Association between the clinicophathological factors and TS, DPD, TP, FPGS, GGH and DHFR mRNA levels.

\begin{tabular}{|c|c|c|c|c|c|c|c|c|c|c|c|c|}
\hline \multirow[b]{2}{*}{ Parameter } & \multicolumn{2}{|c|}{$\mathrm{TS}$} & \multicolumn{2}{|c|}{ DPD } & \multicolumn{2}{|c|}{$\mathrm{TP}$} & \multicolumn{2}{|c|}{ FPGS } & \multicolumn{2}{|c|}{ GGH } & \multicolumn{2}{|c|}{ DHFR } \\
\hline & Median & P-value & Median & P-value & Median & P-value & Median & P-value & Median & P-value & Median & $\mathrm{P}$-value \\
\hline \multicolumn{13}{|l|}{ Age, years } \\
\hline$<65$ & 4.33 & 0.664 & 0.27 & 0.631 & 2.15 & 0.632 & 0.56 & 0.112 & 14.7 & 0.228 & 5.25 & 0.811 \\
\hline$\geq 65$ & 3.91 & & 0.30 & & 2.84 & & 0.62 & & 10.6 & & 5.69 & \\
\hline \multicolumn{13}{|l|}{ Gender } \\
\hline Male & 4.11 & 0.678 & 0.29 & 0.354 & 2.49 & 0.315 & 0.57 & 0.167 & 12.6 & 0.839 & 5.18 & 0.150 \\
\hline Female & 3.95 & & 0.32 & & 3.58 & & 0.65 & & 14.4 & & 6.39 & \\
\hline \multicolumn{13}{|l|}{ Primary site } \\
\hline Colon/rectosigmoid & 4.01 & 0.782 & 0.32 & 0.459 & 2.75 & 0.813 & 0.59 & 0.502 & 12.3 & 0.093 & 5.38 & 0.441 \\
\hline Rectum & 4.24 & & 0.24 & & 2.46 & & 0.60 & & 23.1 & & 5.35 & \\
\hline \multicolumn{13}{|l|}{ Invasion depth } \\
\hline MP & 4.08 & 0.990 & 0.40 & 0.846 & 2.93 & 0.651 & 0.76 & 0.631 & 21.9 & 0.417 & 6.27 & 0.885 \\
\hline SM & 3.95 & & 0.27 & & 2.14 & & 0.35 & & 5.46 & & 6.39 & \\
\hline SS & 4.86 & & 0.24 & & 2.11 & & 0.56 & & 12.2 & & 5.31 & \\
\hline SE & 3.79 & & 0.32 & & 2.96 & & 0.60 & & 13.7 & & 5.22 & \\
\hline SI & 5.77 & & 0.41 & & 3.75 & & 0.50 & & 9.87 & & 5.96 & \\
\hline A & 4.11 & & 0.25 & & 2.71 & & 0.62 & & 23.0 & & 4.77 & \\
\hline \multicolumn{13}{|l|}{ Lymph node metastasis } \\
\hline Node(-) & 4.91 & 0.293 & 0.20 & 0.172 & 2.20 & 0.198 & 0.58 & 0.563 & 10.2 & 0.229 & 5.06 & 0.820 \\
\hline $\operatorname{Node}(+)$ & 3.94 & & 0.32 & & 2.85 & & 0.59 & & 15.6 & & 5.38 & \\
\hline
\end{tabular}

mRNA expression is relative to $\beta$-actin expression levels. TS, thymidylate synthase; DPD, dihydropyrimidine dehydrogenase; TP, thymidine phosphorylase; FPGS, folypolyglutamate synthetase; GGH, $\gamma$-glutamyl hydrolase; DHFR, dihydrofolate reductase; MP, muscularis propria; SM, submucosa; SS, subserosa; SE, serosal exposure; SI, serosal invasion; A, adventitia.

Table IV. Association between DFS and TS, DPD, TP, FPGS, GGH and DHFR mRNA levels.

\begin{tabular}{|c|c|c|c|c|c|c|}
\hline \multirow[b]{2}{*}{ Molecular marker } & \multicolumn{3}{|c|}{ All patients } & \multicolumn{3}{|c|}{ Stage III } \\
\hline & $\mathrm{n}$ & 3-year DFS, \% & P-value & $\mathrm{n}$ & 3-year DFS, \% & P-value \\
\hline \multicolumn{7}{|l|}{ TS } \\
\hline High $(\geq 4.075)$ & 27 & 85.2 & 0.847 & 17 & 88.2 & 0.628 \\
\hline Low $(<4.075)$ & 27 & 85.2 & & 21 & 81.0 & \\
\hline \multicolumn{7}{|l|}{ DPD } \\
\hline High $(\geq 0.295)$ & 27 & 92.6 & 0.310 & 21 & 90.1 & 0.272 \\
\hline Low $(<0.295)$ & 27 & 77.3 & & 17 & 75.3 & \\
\hline \multicolumn{7}{|l|}{$\mathrm{TP}$} \\
\hline High $(\geq 2.575)$ & 27 & 96.3 & 0.066 & 21 & 95.2 & 0.039 \\
\hline Low $(<2.575)$ & 27 & 73.6 & & 17 & 69.3 & \\
\hline \multicolumn{7}{|l|}{ FPGS } \\
\hline High $(\geq 0.590)$ & 27 & 88.9 & 0.313 & 20 & 90.0 & 0.283 \\
\hline Low $(<0.590)$ & 27 & 81.2 & & 18 & 77.0 & \\
\hline \multicolumn{7}{|l|}{ GGH } \\
\hline High $(\geq 12.96)$ & 27 & 88.7 & 0.311 & 21 & 85.5 & 0.803 \\
\hline Low $(<12.96)$ & 27 & 81.5 & & 17 & 82.4 & \\
\hline \multicolumn{7}{|l|}{ DHFR } \\
\hline High $(\geq 5.380)$ & 27 & 81.5 & 0.636 & 19 & 80.0 & 0.338 \\
\hline Low $(<5.380)$ & 27 & 88.6 & & 19 & 88.9 & \\
\hline
\end{tabular}

mRNA expression is relative to $\beta$-actin expression levels. DFS, disease-free survival; TS, thymidylate synthase; DPD, dihydropyrimidine dehydrogenase; TP, thymidine phosphorylase; FPGS, folypolyglutamate synthetase; GGH, $\gamma$-glutamyl hydrolase; DHFR, dihydrofolate reductase. 
colon or rectosigmoid colon in 40 patients $(74.1 \%)$ and in the rectum in 14 (25.9\%). Overall, 16 patients (29.6\%) exhibited stage II disease and 38 (70.4\%) exhibited stage III disease.

Clinicopathological factors versus TS, DPD, TP, FPGS, GGH and DHFR mRNA levels. The mRNA expression levels [median (range)] of TS, DPD, FPGS, GGH and DHFR are shown in Table II. There was no correlation between the mRNA expression levels of any of these enzymes and any of the clinicopathological factors of age, gender, primary site, location, invasion depth or lymph-node metastasis (Table III).

A correlation analysis of TS, DPD and TP, three enzymes involved in 5-FU metabolism, showed a significant positive correlation between TP and DPD (data not shown; Spearman's correlation coefficient, 0.78; $\mathrm{P}<0.0001)$.

DFS versus mRNA levels of TS, DPD, TP, FPGS, GGH and $D H F R$. DFS did not differ significantly between the patients with high mRNA expression and those with low mRNA expression of any factor associated with the sensitivity to various types of anticancer agents in the study group as a whole, but there was a trend toward a longer DFS in the patients with high TP expression $(\mathrm{P}=0.066)$. According to disease stage, no factor was associated with survival in the patients with stage II disease. However, in the patients with stage III disease who received post-operative adjuvant chemotherapy, there was a statistical difference between the association of low TP expression levels and DFS compared with high TP expression levels ( $\mathrm{P}=0.039)$ (Fig. 1). DFS did not differ significantly according to the expression level of any other factor (Table IV).

\section{Discussion}

S-1, an oral 5-FU-based anticancer drug, is indicated for the treatment of seven types of cancer in Japan, including gastric cancer, colorectal cancer, and head and neck cancer (15). S-1 is also approved in various countries in Asia and Europe. S-1 has been found to be at least as effective as conventional 5-FU-based anticancer agents and was designed to reduce gastrointestinal toxicity, an adverse reaction specifically associated with 5-FU analogues. S-1 contains gimeracil, which strongly inhibits DPD, a metabolizing enzyme of 5-FU derivatives, thereby maintaining high concentrations of 5-FU in serum (16). In addition, S-1 contains oteracil potassium, which inhibits the phosphorylation of 5-FU in the gastrointestinal tract, an important cause of gastrointestinal toxicity, and thereby inhibits adverse effects (17). Our previous study analyzed the safety and effectiveness of a one-year treatment with S-1 in patients with resected stage II or III colorectal cancer. The treatment completion rate was $77.7 \%$, and watery eyes was the only grade 3 or higher adverse reaction (1 patient). The three-year DFS rate was $85 \%$, showing that S-1 is safe and effective (9). At present, the usefulness of S-1 as a post-operative adjuvant chemotherapy is being evaluated in phase III clinical trials in patients with colorectal cancer, and S-1 may become a standard treatment for colorectal cancer in the future (18).

The present study measured the mRNA expression levels of TS, DPD, TP, FPGS, GGH and DHFR, enzymes that are important in the chemotherapy of colorectal cancer with 5-FU-based agents, and examined the associations between such levels and DFS. TS, an enzyme required for DNA synthesis, is a target enzyme of 5-FU. DPD is an enzyme that affects the pharmacokinetics of 5-FU. TP is not only involved in 5-FU metabolism, but is also known as a platelet-derived endothelial cell growth factor, which has angiogenic activity (19-21). Several studies have demonstrated that tumors with low levels of TS, DPD and TP gene expression are more sensitive to 5-FU, not only in advanced or recurrent colorectal cancer, but also in gastric cancer and breast cancer (22-25). In particular, TP expression levels have been shown to differ by a factor of 2.6 times between patients who are more sensitive and those who are less sensitive to chemotherapy (22).

Few studies have examined the correlations between TP expression and the clinical usefulness of post-operative adjuvant chemotherapy in patients with colorectal cancer. Sadahiro et al (26) found that post-operative adjuvant chemotherapy with uracil and tegafur (UFT)/leucovorin is beneficial in patients with colorectal cancer and high TP expression levels, and reported that TP expression levels may be a useful predictor of treatment response. Another study showed that high TP expression was associated with a significantly higher survival rate in patients with Duke's C colorectal cancer who received 5'-deoxy-5-fluorouridine (5'-DFUR) (27). Since TP is an enzyme that not only participates in 5-FU metabolism, but also converts 5'-DFUR to 5-FU, it was proposed as a potential predictor of response. By contrast, experimental studies also reported that high TP expression is associated with the decreased sensitivity of colorectal cancer to 5-FU $(28,29)$, and certain clinical trials found no clinically useful correlation between TP expression and the response to post-operative adjuvant chemotherapy with agents such as 5-FU/leucovorin and 5'-DFUR $(30,31)$. The ability to use TP mRNA expression to predict response to post-operative adjuvant chemotherapy in patients with colorectal cancer thus remains controversial.

In the present study, high TP expression was associated with good outcomes, particularly in the patients with stage III disease. These findings and the results of a previous study by Sadahiro et al (26) showing that high TP expression is associated with good outcomes in patients who received UFT/leucovorin suggest that the mechanism of action and clinical effects of post-operative adjuvant chemotherapy with S-1, containing uracil and gimeracil, which prevents 5-FU catabolism by inhibiting DPD, or with regimens that include UFT, differ from those of other 5-FU-based anticancer agents (26). As S-1 and UFT enhance serum 5-FU concentrations by inhibiting DPD, the response to these drugs may be more susceptible to catalytic reactions mediated by TP than other 5-FU analogues.

The present results demonstrated a significant positive correlation between TP and DPD expression. This finding was consistent with the result of a study by Collie-Duguid et al (32), which reported a positive correlation between TP and DPD expression in colorectal cancer. In the present study, however, outcomes similar to those in patients with high TP expression were not obtained in patients with high DPD expression. One of the reasons for 
this finding may be that $\mathrm{S}-1$ was clinically effective regardless of DPD expression.

In conclusion, the present study measured the mRNA expression levels of factors associated with the sensitivity to various types of anticancer agents and found that TP is a predictor of response. The results suggest that TP can be used to predict the response to post-operative adjuvant chemotherapy with S-1. However, as the number of patients was small, firm conclusions could not be drawn. Further large clinical studies of factors associated with sensitivity to various types of anticancer agents are required to confirm these findings.

\section{References}

1. Shirasaka T, Shimamato Y, Ohshimo H, et al: Development of a novel form of an oral 5-fluorouracil derivative (S-1) directed to the potentiation of the tumor selective cytotoxicity of 5-fluorouracil by two biochemical modulators. Anticancer Drugs 7: 548-557, 1996.

2. Diasio RB: Clinical implications of dihydropyrimidine dehydrogenase inhibition. Oncology (Williston Park) 13 (Suppl 3): 17-21, 1999.

3. Yamada Y, Hamaguchi T, Goto M, et al: Plasma concentrations of 5-fluorouracil and F-beta-alanine following oral administration of $\mathrm{S}-1$, a dihydropyrimidine dehydrogenase inhibitory fluoropyrimidine, as compared with protracted venous infusion of 5-fluorouracil. Br J Cancer 89: 816-820, 2003.

4. Ohtsu A, Baba H, Sakata Y, et al: Phase II study of S-1, a novel oral fluoropyrimidine derivative, in patients with metastatic colorectal carcinoma. S-1 Cooperative Colorectal Carcinoma Study Group. Br J Cancer 83: 141-145, 2000.

5. Shirao K, Ohtsu A, Takada H, et al: Phase II study of oral S-1 for treatment of metastatic colorectal carcinoma. Cancer 100: 2355-2361, 2004.

6. Muro K, Boku N, Shimada Y, et al: Irinotecan plus S-1 (IRIS) versus fluorouracil and folinic acid plus irinotecan (FOLFIRI) as second-line chemotherapy for metastatic colorectal cancer: a randomised phase $2 / 3$ non-inferiority study (FIRIS study). Lancet Oncol 11: 853-860, 2010.

7. Sakuramoto S, Sasako M, Yamaguchi T, et al; ACTS-GC Group: Adjuvant chemotherapy for gastric cancer with S-1, an oral fluoropyrimidine. N Engl J Med 357: 1810-1820, 2007.

8. Sasako M, Sakuramoto S, Katai H, et al: Five-year outcomes of a randomized phase III trial comparing adjuvant chemotherapy with S-1 versus surgery alone in stage II or III gastric cancer. J Clin Oncol 29: 4387-4393, 2011.

9. Ogawa M, Watanabe M, Kobayashi T, et al: Feasibility study of S-1 adjuvant chemotherapy in patients with colorectal cancer. Int J Clin Oncol 18: 678-683, 2013.

10. Salonga D, Danenberg KD, Johnson M, et al: Colorectal tumors responding to 5-fluorouracil have low gene expression levels of dihydropyrimidine dehydrogenase, thymidylate synthase, and thymidine phosphorylase. Clin Cancer Res 6: 1322-1327, 2000.

11. Popat S, Matakidou A and Houlston RS: Thymidylate synthase expression and prognosis in colorectal cancer: a systematic review and meta-analysis. J Clin Oncol 22: 529-536, 2004.

12. Sobin LH and Wittekind Ch (eds): TNM Classification of Malignant Tumors. 6th edition. Wiley-Liss, Inc., New York, NY, 2002 .

13. Bonner RF, Emmert-Buck M, Cole K, et al: Laser capture microdissection: molecular analysis of tissue. Science 278: 1481, 1997.

14. Lord RV, Salonga D, Danenberg KD, et al: Telomerase reverse transcriptase expression is increased early in the Barrett's metaplasia, dysplasia, adenocarcinoma sequence. J Gastrointest Surg 4: 135-142, 2000.
15. Satoh T and Sakata Y: S-1 for the treatment of gastrointestinal cancer. Expert Opin Pharmacother 13: 1943-1959, 2012.

16. Shirasaka T, Shimamoto Y and Fukushima M: Inhibition by oxonic acid of gastrointestinal toxicity of 5-fluorouracil without loss of its antitumor activity in rats. Cancer Res 53: 4004-4009, 1993

17. Shirasaka T, Shimamato Y, Ohshimo H, et al: Development of a novel form of an oral 5-fluorouracil derivative (S-1) directed to the potentiation of the tumor selective cytotoxicity of 5-fluorouracil by two biochemical modulators. Anticancer Drugs 7: 548-557, 1996

18. Mochizuki I, Takiuchi H, Ikejiri K, et al: Safety of UFT/LV and $\mathrm{S}-1$ as adjuvant therapy for stage III colon cancer in phase III trial: ACTS-CC trial. Br J Cancer 106: 1268-1273, 2012.

19. Furukawa T, Yoshimura A, Sumizawa T, et al: Angiogenic factor. Nature 356: 668, 1992.

20. Takebayashi Y, Akiyama S, Akiba S, et al: Clinicopathologic and prognostic significance of an angiogenic factor, thymidine phosphorylase, in human colorectal carcinoma. J Natl Cancer Inst 88: 1110-1117, 1996.

21. Nakayama Y, Inoue $\mathrm{Y}$, Nagashima $\mathrm{N}$, et al: Expression levels of thymidine phosphorylase (TP) and dihydropyrimidine dehydrogenase (DPD) in patients with gastrointestinal cancer. Anticancer Res 25: 3755-3761, 2005.

22. Metzger R, Danenberg K, Leichman CG, et al: High basal level gene expression of thymidine phosphorylase (platelet-derived endothelial cell growth factor) in colorectal tumors is associated with nonresponse to 5-fluorouracil. Clin Cancer Res 4: 2371-2376, 1998.

23. Toi M, Hoshina S, Taniguchi $\mathrm{T}$, et al: Expression of platelet-derived endothelial cell growth factor/thymidine phosphorylase in human breast cancer. Int J Cancer 64: 79-82, 1995.

24. Takebayashi Y, Akiyama S, Akiba, S, et al: Clinicopathologic and prognostic significance of an angiogenic factor, thymidine phosphorylase, in human coborectal carcinoma. J Natl Cancer Inst 88: 1110-1117, 1996.

25. Maeda K, Chung YS, Ogawa Y, et al: Thymidine phosphorylase/platelet-derived endothelial cell growth factor expression associated with hepatic metastasis in gastric carcinoma. Br J Cancer 73: 884-888, 1996.

26. Sadahiro S, Suzuki T, Tanaka A, Okada K, Nagase H and Uchida $\mathrm{J}$ : Association of right-sided tumors with high thymidine phosphorylase gene expression levels and the response to oral uracil and tegafur/leucovorin chemotherapy among patients with colorectal cancer. Cancer Chemother Pharmacol 70: 285-291, 2012.

27. Hasegawa S, Seike K, Koda K, et al: Thymidine phosphorylase expression and efficacy of adjuvant doxifluridine in advanced colorectal cancer patients. Oncol Rep 13: 621-626, 2005.

28. Salonga D, Danenberg KD, Johnson M, et al: Colorectal tumors responding to 5-fluorouracil have low gene expression levels of dihydropyrimidine dehydrogenase, thymidylate synthase, and thymidine phosphorylase. Clin Cancer Res 6: 1322-1327, 2000.

29. Yoshinare K, Kubota T, Watanabe M, et al: Gene expression in colorectal cancer and in vitro chemosensitivity to 5-fluorouracil: a study of 88 surgical specimens. Cancer Sci 94: 633-638, 2003.

30. Nishimura G, Terada I, Kobayashi T, et al: Thymidine phosphorylase and dihydropyrimidine dehydrogenase levels in primary colorectal cancer show a relationship to clinical effects of 5'-deoxy-5-fluorouridine as adjuvant chemotherapy. Oncol Rep 9: 479-482, 2002.

31. Jensen SA, Vainer B, Witton CJ, Jørgensen JT and Sørensen JB: Prognostic significance of numeric aberrations of genes for thymidylate synthase, thymidine phosphorylase and dihydrofolate reductase in colorectal cancer. Acta Oncol 47: 1054-1061, 2008.

32. Collie-Duguid ES, Johnston SJ, Boyce L, et al: Thymidine phosphorylase and dihydropyrimidine dehydrogenase protein expression in colorectal cancer. Int J Cancer 94: 297-301, 2001. 